\title{
Bilateral Humerus Fractures in a New-Born During a Caesare- an Delivery: Case Report and Critical Review of the Literature
}

\author{
Pietro Persiani ${ }^{1 *}$, Marco Bove ${ }^{1}$, Annamaria Dell'Unto ${ }^{1}$, Filippo Maria Ranaldi' ${ }^{1}$ Ilaria Stolfi', Roberto Pedicino ${ }^{3}$, Ciro Villani ${ }^{1}$
}

${ }^{1}$ Department of Anatomical, Histological, Medical-Legal Sciences and Locomotor Apparatus, Umberto I Polyclinic, La Sapienza University of Rome. Piazzale Aldo Moro 3, Rome, Italy

${ }^{2}$ Policlinico Umberto I, La Sapienza University of Rome. Viale del Policlinico 155, Rome, Italy

${ }^{3}$ Department of Pediatrics and Child Neuropsychiatry, Policlinico Umberto I, La Sapienza University of Rome. Viale del Policlinico 155, Rome, Italy

"Corresponding author: Pietro Persiani, Department of Anatomical, Histological, Medical-Legal Sciences and Locomotor Apparatus, Umberto I Polyclinic, La Sapienza University of Rome. Piazzale Aldo Moro 3, 00161 Rome, Italy. Tel: + 39335497510; Email: ppersiani@me.com

Citation: Persiani P, Bove M, Dell'Unto A, Ranaldi FM, Stolfi I, et al. (2018) Bilateral Humerus Fractures in a New-Born During a Caesarean Delivery: Case Report and Critical Review of the Literature. Arch Surg Clin Case Rep: ASCR-109. DOI: 10.29011/ ASCR-109/100009

Received Date: 30 August, 2018; Accepted Date: 11 September, 2018; Published Date: 19 September, 2018

\begin{abstract}
Purpose: To analyse clinical case of neonatal bilateral humerus fractures, which represent a very rare event in births by caesarean section.

Method: We analysed the case of a new-born with a bilateral humerus fractures associated with a caesarean delivery in the Paediatric Intensive Care Unit of our centre. The infant was delivered premature at seven and a half months, weighed 1,100g, was $33.4 \mathrm{~cm}$ in length and was born by caesarean section performed on a primiparous mother in non-urgent conditions. The extraction of the new-born was hampered by the entrapment of the head. The new-born's family history was negative for cases of osteogenesis imperfecta or other osteo-metabolic genetic diseases. The treatment adopted was a partial reduction and immobilization under sedation. The radiographic follow-up performed at 4 weeks showed a bone remodelling with good alignment of the axis. From a clinical point of view, the new-born had neither deficits nor residual deformities.

Results: The main risk factors are: prematurity, low birth weight, foetal malposition, uterine defects, first pregnancy, delivery in conditions of emergency and iatrogenic birth traumas. In these cases, it is important to exclude the possibility of pathologies of an osseous or chromosomal nature.

Conclusion: It is essential to immediately dispel any doubts regarding the possibility of osseous or chromosomal disorders. The careful management of the medical-parent relationship is important in order to best handle parental anxiety and avoid the potential risk of unnecessary medical disputes.
\end{abstract}

Keywords: Bilateral; Caesarean; Fracture; Humerus; Obstetric

\section{Introduction}

Italy has recorded an increase in the rate of caesarean section births. This procedure has risen from $11.2 \%$ in 1980 to $36.3 \%$ in 2013 (compared to the European average of $26.7 \%$ in 2011 and that of the United States and Canada respectively at $31.4 \%$ and $26.1 \%$ in 2010) [1].
The incidence of skeletal injuries in live births during a caesarean section is less than $1 \%[2,3]$. The risk factors that predispose this occurrence are: prematurity, foetal macrosomia, intrauterine malposition or breech position, emergency conditions, iatrogenic causes and uterine defects $[2,3,4]$.

In the literature, the most frequently reported lesions are clavicle fractures and femoral fractures. Fractures of the humerus are extremely rare events, especially bilateral ones $[2,4,5,6]$. 


\section{Case Report}

Here in we report the case of a new-born admitted to the Pediatric Intensive Care Unit of the "Umberto I" University Hospital of Rome with a diagnosis of bilateral humerus fractures after a caesarean section delivery. The patient was premature at 36 weeks, weighed 2500 and was $33.4 \mathrm{~cm}$ in length.

The patient had no family history of osteo-metabolic genetic diseases such as osteogenesis imperfecta. No uterine alteration was observed in the obstetric anamnesis.

As the delivery was complicated by the entrapment of the infant's head, a traction/rotation extraction was performed by gripping the arms and chest of the new-born.

\section{The New-born}

The infant was immediately assessed post-birth and showed no signs of hypoxia, but the upper limbs showed swelling, an angular deviation of $40^{\circ}$ and was incessantly crying.

An X-ray examination was performed confirming bilateral varus fractures of the mid-third diaphyseal of the humerus (Figure 1), type $\mathrm{D} / 5$ according to the AO/ASIF classification [7].

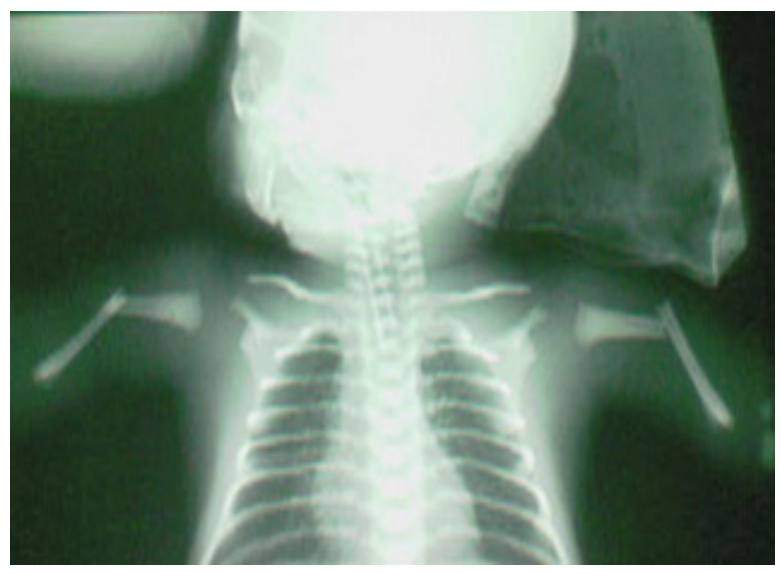

Figure 1: X-ray presentation showing a bilateral diaphyseal fracture within hours of birth.

After excluding possible vascular-nervous deficiencies, a closed reduction was performed, and a Mayo immobilization was applied bilaterally. The new-born was then placed in an incubator for 4 weeks. The immobilization was removed after 28 days. Clinically, the infant's mobility was complete, without pain and there were no functional deficits or deformities.

The x-ray performed at 2 months showed the consolidation of the fractures and a good alignment of the humeral axis bilaterally. (Figures 2 and 3)

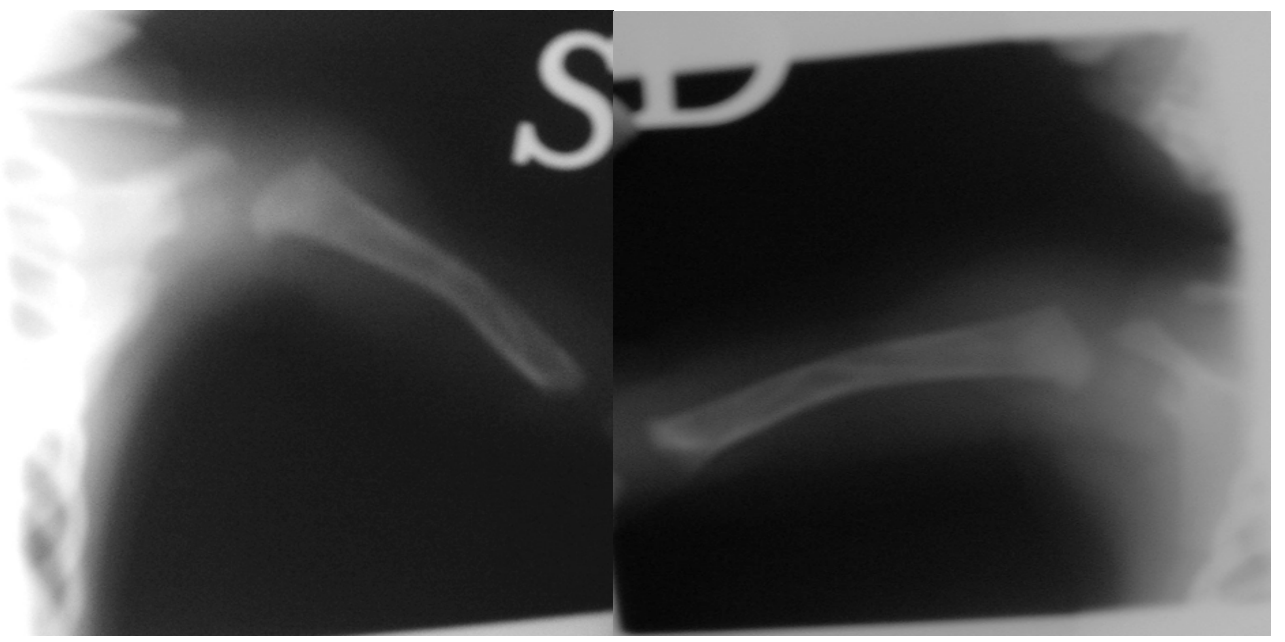

Figure 2: X-ray of the left humerus at 2 months of age. Figure 3: X-ray of the right humerus at 2 months of age.

\section{Discussion}

In the literature, the incidence of fractures in live births during a caesarean section is less than $1 \%[6,7]$. Risk factors and related causes are prematurity, low birth weight, poor foetal positioning, uterine defects, first pregnancy, iatrogenic birth traumas and a delivery under conditions of emergency [2,3,4]. Emergency
Caesarean sections can expose the infant to a greater risk of longbone fractures compared to vaginal births; it is thus fundamental to plan the procedure and avoid dangerous manoeuvres so as to circumvent these risks $[2,6]$.

Clavicle fractures and femur fractures are the most frequent lesions $[2,4,5,6]$. Humerus fractures (especially bilateral ones) are 


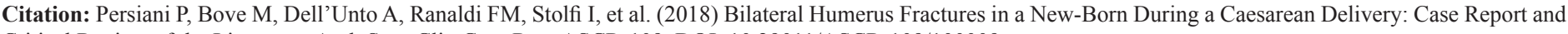
Critical Review of the Literature. Arch Surg Clin Case Rep: ASCR-109. DOI: 10.29011/ASCR-109/100009

an exceptional event, sometimes associated with Braxial Plexus Injury (BPI) [8] , and very rarely associated with caesarean sections $[4,5,6]$.

Once the clinical and instrumental diagnosis has been performed and genetic diseases have been excluded, the treatment of said fractures involves only immobilization and clinical monitoring. The immobilization can be removed after about 4 weeks. Neonatal fractures have a good prognosis [2], with bone callus formation within 8/12 days and consolidation in 4 weeks. It is a well-known fact that new-borns have a great bone remodelling capacity, regardless of the degree of fracture displacement. There are, in fact, no guide lines that indicate what the acceptable fracture angulation in new-borns is [9].

\section{Conclusion}

Bilateral humerus fractures are a very rare event in caesarean section births. When they do occur, it is necessary to exclude any possible congenital osteo-metabolic pathologies. A closed reduction of the fracture guarantees a "restitutio ad integrum", also in the presence of severe displacement or serious angulation. In any case, in the presence of risk factors, the fractures must not be considered as an index of the quality of the caesarean extraction. In these delicate situations, careful and correct management of the doctor-parent relationship is also necessary in order to manage the parent's anxiety and avoid the potential risk of legal medical disputes, especially in light of the excellent clinical and functional results.

\section{References}

1. Report Istat Anno (2013) Gravidanze, parto ed allattamento al seno. www.instat.it

2. Basha A, Amarin Z, Abu-Hassan F (2013) Birth-associated long-bone fractures. International Journal of Gynecology and Obstetrics 123: $127-130$.

3. Morris S, Cassidy N, Stephens M, McCormack D, McManus F (2002) Birth-Associated Femoral Fractures: Incidence and Outcome. Journal of Pediatric Orthopaedics 22: 27-30.

4. Dias E (2012) Bilateral Humerus Fracture Following Birth Trauma. Journal of Clinical Neurology 1: 45-46.

5. Canpolat FE, Köse A, Yurdakök M (2010) Bilateral humerus fracture in a neonate after caesarean delivery. Arch Gynecol Obstet 281: $967-$ 969.

6. Rahul P, Grover AR, Ajoy SM (2017) Bilateral Humerus and Right Femur Fracture in a Newborn after Cesarean Section for Breech Presentation in a Twin Pregnancy: A Very Rare Case Report. Journal of Orthopaedic Case Reports 7: 9-11.

7. Buckley RE, Rüedi TP, Moran CG (2008) AO Principles for the Treatment. In: Vol 1 Fractures - Principles - Part 4 - General Arguments Section 4.4 Loss Surgery. CIC International Editions 2008 (398-402).

8. Tan TS, Mohamed A, Dharmaraj S (2016) Bilateral fractures in a shoulder dystocia delivery. BMJ Case Report 2016.

9. Husain SN, King EC, Young JL, Sarwark JF (2008) Remodeling of birth fractures of the humeral diaphysis. J Pediatr Orthop 28: 10-13. 